Research Article

\title{
Efficacy and Safety of EUS-Guided Choledochoduodenostomy Using Electrocautery-Enhanced Lumen-Apposing Metal Stents (ECE-LAMS) in the Treatment of Biliary Obstruction: A Systematic Review and Meta-Analysis
}

\author{
Zuxiang Peng, Shan Li, Yongliang Tang, Wanjie Wei, Ruxian Pi, Xianchun Liang, \\ Yafeng Wan, and Hongming Liu 1
}

Department of Hepatobiliary Surgery, Daping Hospital, Army Medical University, Chongqing, China

Correspondence should be addressed to Hongming Liu; liuhongming74@163.com

Received 4 November 2020; Revised 10 March 2021; Accepted 19 March 2021; Published 30 March 2021

Academic Editor: Ravindran Caspa Gokulan

Copyright ( 2021 Zuxiang Peng et al. This is an open access article distributed under the Creative Commons Attribution License, which permits unrestricted use, distribution, and reproduction in any medium, provided the original work is properly cited.

Background. Electrocautery-enhanced lumen-apposing metal stents (ECE-LAMS) have been newly developed to perform EUSguided choledochoduodenostomy (EUS-CDS), but its benefits and harms remain obscure. We conducted a systematic review and meta-analysis to evaluate the efficacy and safety of EUS-CDS using ECE-LAMS. Method. In the Preferred Reporting Items for Systematic Reviews and Meta-Analyses (PRISMA), we searched PubMed, Embase, and Scopus databases through January 1, 2001, and April 27, 2020. The primary outcomes of the pooled analysis were to determine the technical success, clinical success, and overall adverse events rates. The secondary outcomes were pooled rates of short-term and long-term adverse events. Results. Six studies with 270 patients were finally included in this meta-analysis. The pooled rates of technical, clinical success, and adverse events were 95.1\% (95\% CI = 90.6-97.5\%, $\left.I^{2}=25 \%\right), 93.3 \%\left(95 \% \mathrm{CI}=87.4-96.5 \%, I^{2}=28 \%\right)$, and $15.3 \%(95 \% \mathrm{CI}=10.6-21.6 \%$, $\left.I^{2}=13 \%\right)$, respectively. The pooled rates of short-term and long-term adverse events were $3.6 \%\left(95 \% \mathrm{CI}=1.3-9.6 \%, I^{2}=0 \%\right)$ and $11.3 \%\left(95 \% \mathrm{CI}=7.6-16.5 \%, I^{2}=0 \%\right)$, respectively. Conclusion. EUS-CDS using ECE-LAMS provides favorable outcomes in patients with biliary obstruction. It has been associated with a higher success rate and a lower rate of adverse events when compared with the biliary drainage approaches previously used. Large and randomized controlled observational studies are required to further refine the findings in the present analysis.

\section{Introduction}

Over the last decades, endoscopic retrograde cholangiopancreatography (ERCP) with stent placement is prominently applied to perform biliary drainage in patients with biliary obstruction [1]. The failure rate is still about $10 \%$, even though this treatment was performed by expert endoscopists [2-4]. When ERCP fails, percutaneous transhepatic biliary drainage (PTBD) and surgical bypass are reliable alternatives. However, such strategies have been associated with relatively high morbidity, prolonged hospitalization, poor life quality, and several drainage-related complications $[5,6]$.
EUS-guided choledochoduodenostomy (EUS-CDS) has emerged as one of the promising techniques for biliary drainage after ERCP failure. In 2001, Giovannini et al. first reported one case of successful EUS-CDS, which places a stent across the duodenal wall into the extrahepatic bile duct, in a patient with malignant biliary obstruction after failed ERCP [7-9]. A recent meta-analysis reported that EUS-CDS had a clinical success rate of $88.5 \%$ and an adverse event rate of $18.6 \%$ [10]. However, use of a plastic stent that initially served for EUS-CDS may result in early stent occlusion, bile leak, and subsequent peritonitis $[11,12]$. More recently, EUS-CDS has been significantly changed with the advent of self-expanding metal stents (SEMSs), which are associated 
with a longer survival, a lower rate of stent dysfunction, and a lower reintervention rate when compared with plastic stents [13]. We should, however, note that SEMSs migration may cause tissue injury, which is its main drawback $[14,15]$.

Intriguingly, electrocautery-enhanced lumen-apposing metal stent (ECE-LAMS) was developed to perform EUSCDS [16]. Such stent reduces bile leakage and improves success rates. Nevertheless, to the best of our knowledge, it is unclear whether the EUS-CDS with ECE-LAMS is more appropriate than other drainage approaches for patients who had biliary obstruction. In the present study, we evaluate the efficacy and safety of EUS-CDS with ECE-LAMS by performing a comprehensive systematic review and metaanalysis.

\section{Methods}

2.1. Search Strategy. This systematic review and metaanalysis have been designed and conducted following the guidelines of the Preferred Reporting Items for Systematic Reviews and Meta-Analyses (PRISMA) [17]. We searched PubMed, Embase, and Scopus databases through January 1, 2001, and April 27, 2020, for studies regarding biliary drainage with EUS-CDS. The following search terms were used: "endoscopic ultrasound, EUS, lumen-apposing metal stents, LAMS, lumen-apposing fully covered metal stent, lumen-apposing stents, choledochoduodenostomy, CDS, transmural biliary drainage, EUS-guided choledochoduodenostomy, and EUS-CDS." Manual searches for reference lists of retrieved articles from published literatures were also performed. The search was restricted to studies on humans, which were published only in English. Only published data are retrieved in this meta-analysis. Ethical approval was not required as the study is a systematic review and meta-analysis.

2.2. Inclusion and Exclusion Criteria. After removal of duplicates, two authors (Z.P. and S.L.) independently screened titles and abstracts. To avoid duplicate or overlap results retrieved from the same study cohort, only the most recent study and/or the publication presenting the largest datasets was included in further analysis. We included studies investigating (1) EUS-CDS using ECE-LAMS; (2) outcome measures (technical success rate, clinical success rate, and adverse events); (3) a sample size of more than ten patients. We excluded studies investigating (1) EUS-CDS using LAMS; (2) EUS-guided drainage of pancreatic fluid collections using LAMS; (3) EUS-guided gallbladder drainage with LAMS. Case reports, reviews, letters, comments, and editorials were also excluded. Any differences were resolved by discussion. We retrieved the full text for further evaluation if it seemed to meet the eligibility criteria.

2.3. Data Extraction. Two authors (Z.P. and S.L.) independently extracted information from the original articles using a predetermined data extraction sheet, with discrepancies resolved by discussion. If any disagreement persisted, the final decision was made by a third author (Y.T.) after reviewing the original articles. The study details included study characteristics (first author, country, year of publication, study design, and follow-up period), study population (total number of patients analyzed, patient demographics, etiology, stent diameter, and causes of ERCP failure), and outcome (technical success rate, clinical success rate, and details of adverse events).

2.4. Assessment of Outcome. The primary outcomes assessed in this meta-analysis were the pooled technical success, clinical success, and overall adverse event rates. The secondary outcomes were the pooled rates of short-term and long-term adverse events associated with EUS-CDS using ECE-LAMS.

We followed the definitions of technical and clinical success as defined by individual studies. According to the American Society for Gastrointestinal Endoscopy lexicon [18], short-term adverse event was defined as all complications occurring within 14 days after stent placement. Long-term adverse event was defined as any event occurring 14 days after placement.

2.5. Statistical Analysis. Continuous variables were presented as means (standard deviation, SD) or medians (interquartile range, IQR). Categorical variables were presented as numbers and proportions. We calculated the pooled technical success rate, clinical success rate, and incidence of adverse events and 95\% confidence intervals (CIs). These were analyzed and pooled using the randomeffects model [19]. Heterogeneity was assessed using $I^{2}$ statistics $[20,21]$. The $I^{2}$ values $<30 \%, 30 \%$ to $60 \%, 61 \%$ to $75 \%$, and $>75 \%$ were suggestive of low, moderate, substantial, and considerable heterogeneity, respectively [22]. In all cases, $p<0.05$ was considered statistically significant. We used $\mathrm{R}$ software ( $\mathrm{R}$ version 4.0.2) for all analyses.

\section{Results}

3.1. Characteristics of Included Studies. We collected 247 records from PubMed, 355 records from Scopus, and 549 records from Embase in the primary search and removed 448 duplicates from the initial 1,151 records. Following the inspection of titles and abstracts, 20 studies were selected for full-text assessment. Six studies were included in the final analysis [23-28]. Figure 1 shows the flow diagram for study selection. Four studies [25-28] were multicenter, and the other two $[23,24]$ were single-center studies. Two studies $[26,28]$ were prospective, and the others were retrospective cohort studies. All studies were published between 2018 and 2020. Two studies were conducted in France [26, 27], one in Asia [28], two in Italy [23, 24], and one in North America [25]. Four studies [23, 26-28] used the American Society for Gastrointestinal Endoscopy lexicon for grading of adverse events [18], whereas the other two were defined individually. In most of the studies, technical success was defined as a successful ECE-LAMS deployment between the bile duct and duodenal lumen, and clinical success was defined as a reduction of at least $50 \%$ in total serum bilirubin levels. 


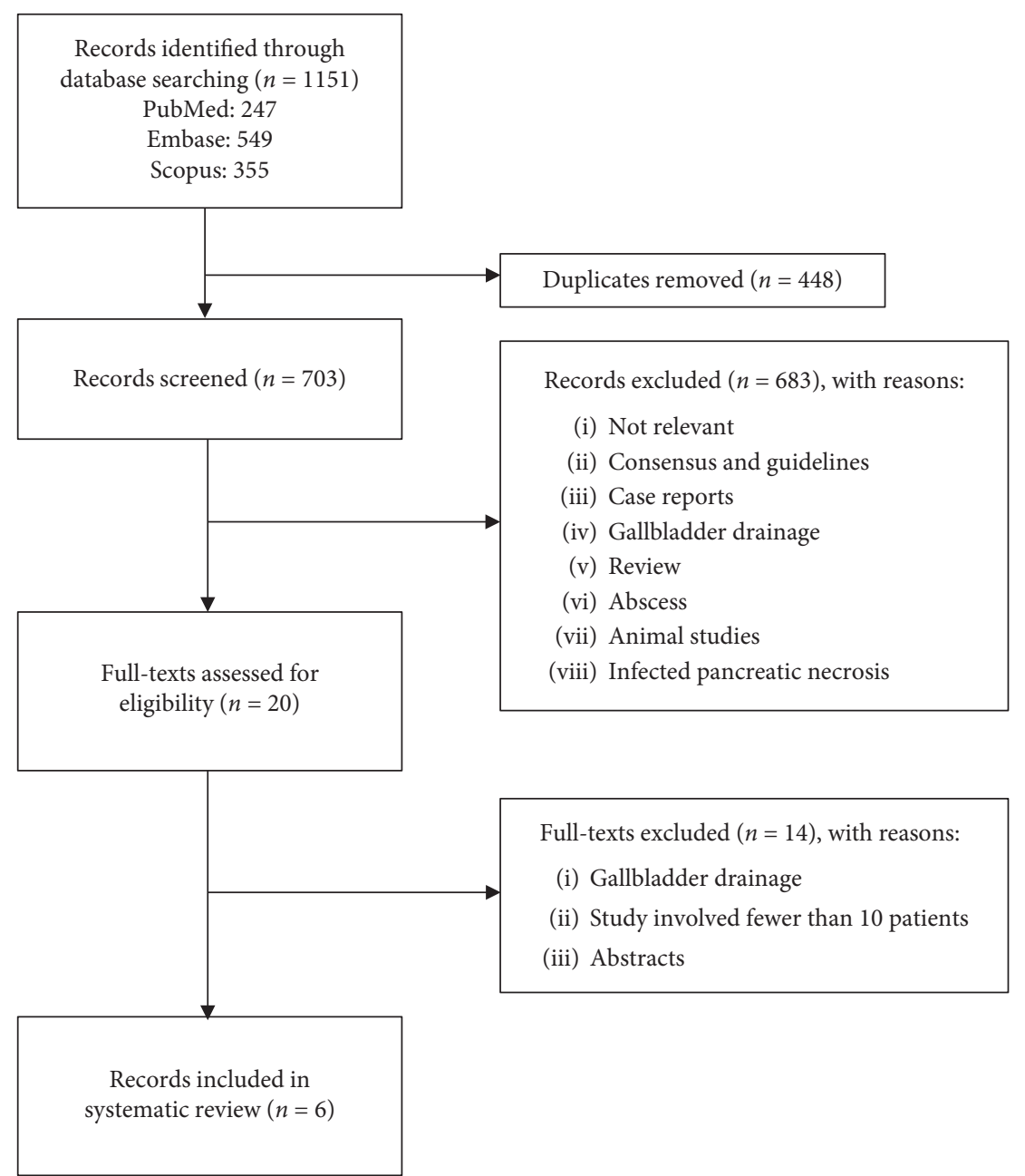

Figure 1: Flow diagram of literature search and selection.

A total of 270 patients were included in the analysis, comprising 144 men (53.3\%) and 126 women (46.7\%), and the mean age ranged from 69.9 to 78 years. The most common cases, in a descend trend, were pancreatic cancer (54.4\%), ampullary cancer, metastatic cancer, and cholangiocarcinoma. Five studies [23, 25-28] reported the mean diameter of common bile duct (CBD), ranging from 17.2 to $17.7 \mathrm{~mm}$. Duodenal stenosis was the most common causes of ERCP failure (77/270). All the included studies had detailed follow-up information. The median follow-up period ranged from 83 to 157 days, and 20 patients were lost to follow-up. In most of the studies, the selection of stent size depended on the CBD diameter but was ultimately at the discretion of the endosonographer. In all the studies, $6 \mathrm{~mm}, 8 \mathrm{~mm}, 10 \mathrm{~mm}$, and $15 \mathrm{~mm}$ stents were used in 143 (53.0\%), 48 (17.8\%), 77 (28.5\%), and $2(0.7 \%)$ cases, respectively. In all included studies, the ECE-LAMS was manufactured by Boston Scientific Corporation. Table 1 shows the characteristics of the studies.

3.2. Efficacy of EUS-CDS with ECE-LAMS. All included studies reported technical success rates. The pooled rate of technical success was $95.1 \%\left(95 \% \mathrm{CI}=90.6-97.5 \% ; I^{2}=25 \%\right)$
(Figure 2(a)). Five studies [23, 24, 26-28] were included in the final analysis of the clinical success rate; one study was excluded since the clinical success was not assessed in 35.8\% of the patients (24/67) [25]. The pooled clinical success rate was $93.3 \%\left(95 \% \mathrm{CI}=87.4-96.5 \% ; I^{2}=28 \%\right)$ (Figure $2(\mathrm{~b})$ ).

3.3. Adverse Events of EUS-CDS with ECE-LAMS. Due to the high rate of lost to follow-up, El Chafic et al. [25] was excluded from the analysis of adverse event rates. Of the 203 patients included in the final analysis, adverse events associated with EUS-CDS using ECE-LAMS were reported in 31 patients $\left(15.3 \%, 95 \% \mathrm{CI}=10.6-21.6 \%, I^{2}=13 \%\right)$ (Figure 2(c)). In the pooled patient population, the percentage of short-term adverse events was 3.6\% (95\% CI $\left.=1.3-9.6 \%, I^{2}=0 \%\right)$ (Figure 2(d)). The most common short-term adverse event was cholangitis (2.0\%). The pooled rate of long-term adverse events was 11.3\% (95\% $\mathrm{CI}=7.6-16.5 \%, I^{2}=0 \%$ ) (Figure $2(\mathrm{e})$ ). The most frequently reported long-term adverse events were tumoral obstruction of the stent $(n=12)$, stent migration $(n=3)$, food residue $(n=2)$, bleeding $(n=2)$, and sump syndrome $(n=2)$. The median time from ECE-LAMS placement to onset of stent obstruction due to tumoral invasion was 130 days (range: 44-282) (Table 2). 


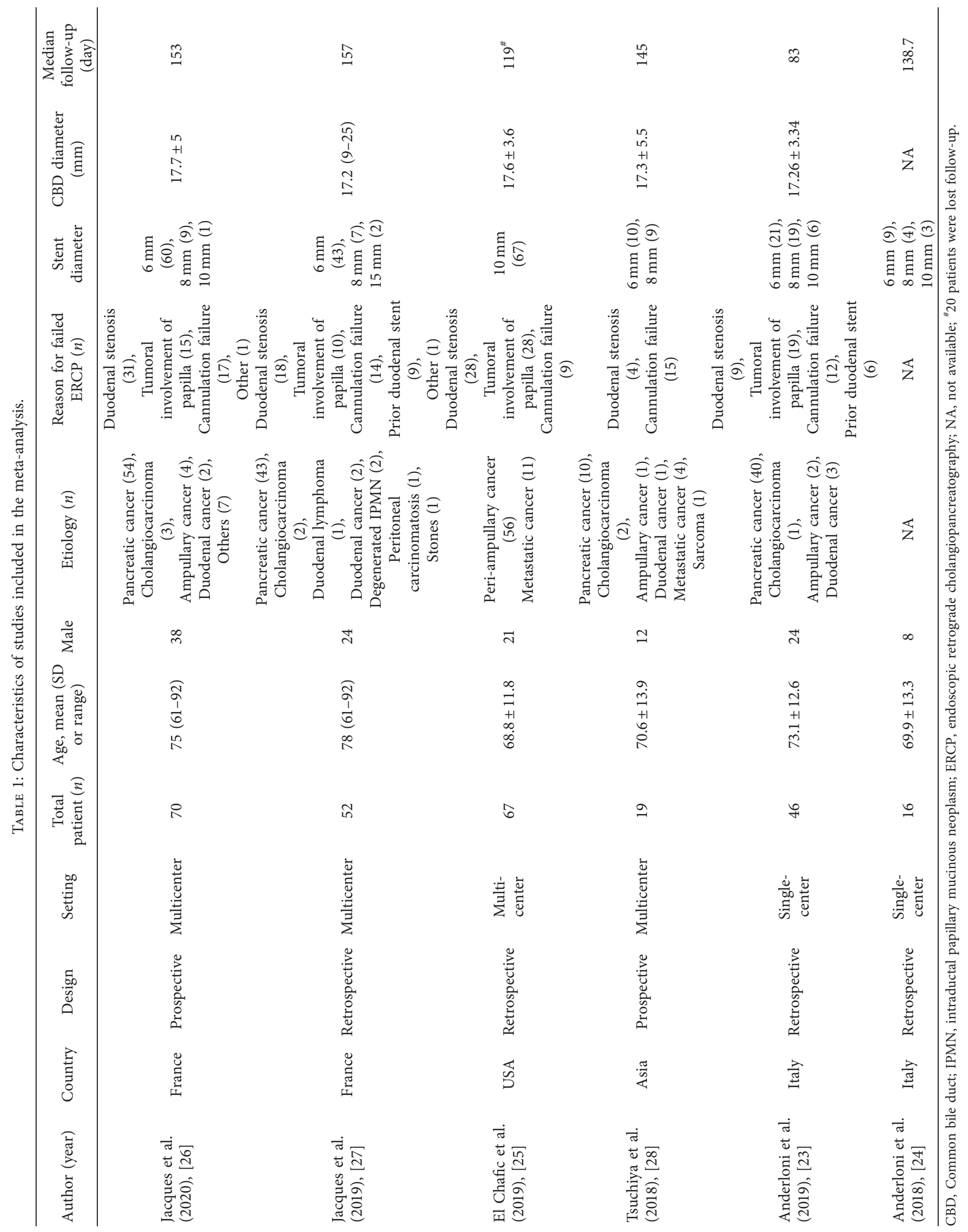




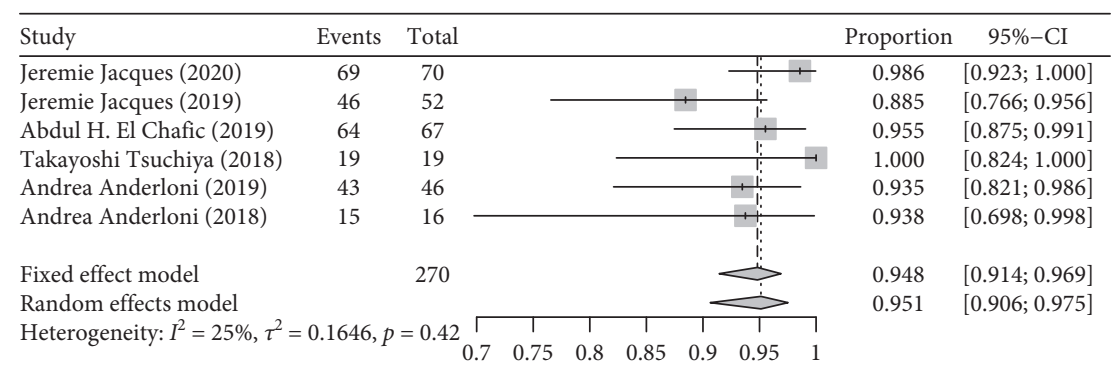

(a)

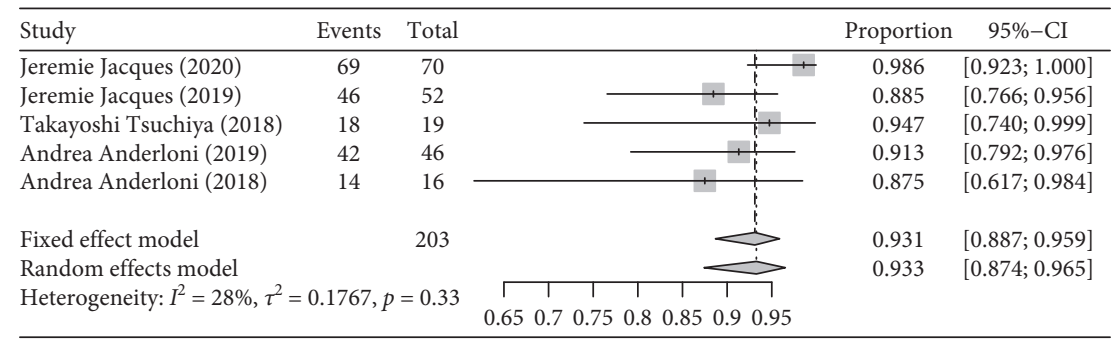

(b)

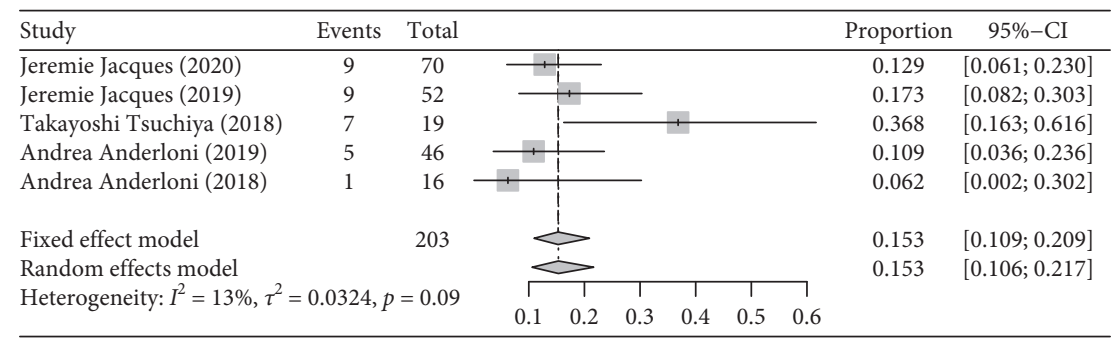

(c)

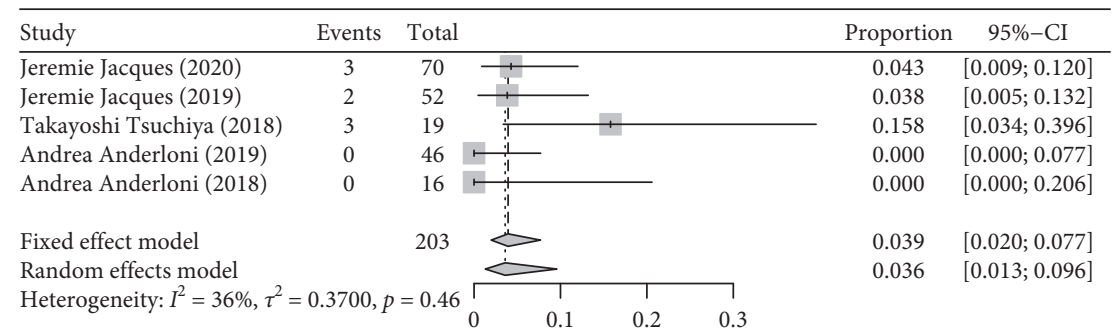

(d)

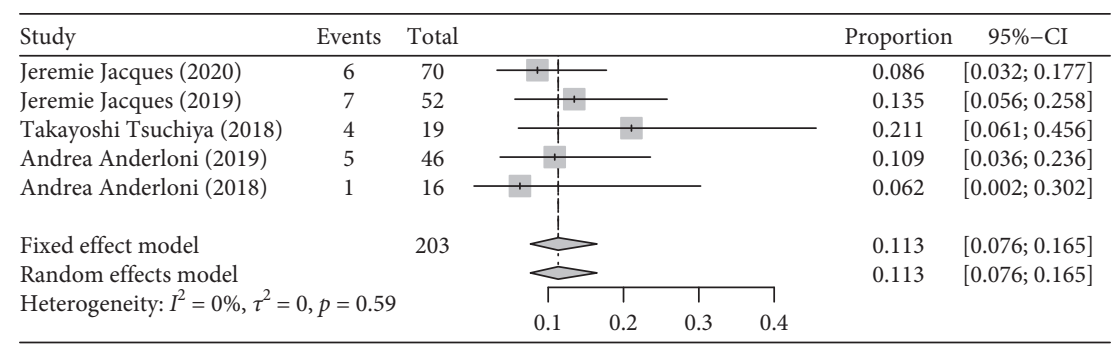

(e)

Figure 2: Forest plot clinical outcomes. (a) Technical success rate. (b) Clinical success rate. (c) Overall adverse event rate. (d) Short-term adverse event rate. (e) Long-term adverse event rate. 
TABLE 2: Outcomes from studies included in the meta-analysis.

\begin{tabular}{|c|c|c|c|c|c|c|}
\hline Author (year) & $\begin{array}{c}\text { Total } \\
\text { patient }(n)\end{array}$ & $\begin{array}{c}\text { Technical } \\
\text { success }(n)\end{array}$ & $\begin{array}{c}\text { Clinical } \\
\text { success }(n)\end{array}$ & $\begin{array}{c}\text { Overall adverse } \\
\text { events }(n)\end{array}$ & $\begin{array}{l}\text { Short-term adverse } \\
\text { events }(n)\end{array}$ & $\begin{array}{c}\text { Long-term adverse } \\
\text { events }(n)\end{array}$ \\
\hline $\begin{array}{l}\text { Jacques et al. (2020), } \\
\text { [26] }\end{array}$ & 70 & 69 & 69 & 9 & $\begin{array}{c}\text { Bleeding (1), } \\
\text { Technical success was } \\
\text { not achieved (1), } \\
\text { Cholangitis (1) }\end{array}$ & $\begin{array}{c}\text { Tumoral obstruction } \\
(4), \\
\text { Stent migration (1), } \\
\text { Bacteremia (1) }\end{array}$ \\
\hline $\begin{array}{l}\text { Jeremie Jacques } \\
(2018),[27]\end{array}$ & 52 & 46 & 46 & 9 & $\begin{array}{l}\text { Bleeding (1), } \\
\text { Cholangitis (1) }\end{array}$ & $\begin{array}{c}\text { Tumoral } \\
\text { obstruction }(4)^{\mathrm{a}}, \\
\text { Sump syndrome }(2)^{\mathrm{a}} \text {, } \\
\text { Stent migration }(1)\end{array}$ \\
\hline $\begin{array}{l}\text { El Chafic (2019), } \\
\text { [25] }\end{array}$ & 67 & 64 & NA & NA & NA & NA \\
\hline $\begin{array}{l}\text { Tsuchiya (2018), } \\
{[28]}\end{array}$ & 19 & 19 & 18 & 7 & $\begin{array}{c}\text { Cholangitis }(2)^{\mathrm{b}}, \\
\text { Fever }(1)\end{array}$ & $\begin{array}{l}\text { Food residue (1), } \\
\text { Stent kinking (1), } \\
\text { Tumor obstruction (1) } \\
\text { Stent migration (1) }\end{array}$ \\
\hline $\begin{array}{l}\text { Anderloni (2018), } \\
{[23]}\end{array}$ & 46 & 43 & 42 & 5 & 0 & $\begin{array}{c}\text { Bleeding (1), } \\
\text { Tumoral obstruction } \\
\text { (3), } \\
\text { Food residue (1) }\end{array}$ \\
\hline $\begin{array}{l}\text { Andrea Anderloni } \\
(2018),[24]\end{array}$ & 16 & 15 & 14 & 1 & 0 & Bleeding (1) \\
\hline
\end{tabular}

${ }^{\mathrm{a}}$ Recurrence of jaundice with symptoms of cholangitis; ${ }^{\mathrm{b}}$ one patient underwent cholangitis because of food residue. NA, not available.

\section{Discussion}

LAMS was originally designed for EUS-guided drainage of pancreatic fluid collections [29-31]. In 2014, Itoi et al. firstly reported one case of successful EUS-CDS with LAMS for biliary drainage after ERCP failure in a patient with unresectable pancreatic cancer [32]. The EUS-CDS using LAMS or ECE-LAMS has been gradually performed for biliary drainage. However, the risk and success rates of EUS-CDS using ECE-LAMS remain obscure. The present meta-analysis evaluated the efficacy and safety of EUS-CDS with ECELAMS. We observed that pooled rates of technical success, pooled rates of clinical success, and overall adverse events following EUS-CDS with ECE-LAMS were 95.1\%, 93.3\%, and $15.3 \%$, respectively. Moreover, pooled rates of shortterm adverse events and long-term adverse events of ECELAMS were $3.6 \%$ and $11.3 \%$, respectively.

Over the past decade, EUS-CDS has been recognized as an alternative approach when ERCP fails, and its role as a first-line treatment for malignant biliary obstruction has also become increasingly recognized [33, 34]. A recent meta-analysis, which included three randomized trials conducted in the USA and Korea [35-37], compared the efficacy and safety between EUS-CDS with SEMS and ERCP-BD that serve for primary palliation of malignant biliary obstruction [38]. Though there was no difference between the approaches regarding the overall adverse events, clinical success rates, and occlusion rates, EUS-CDS using SEMS was associated with lower rate of postprocedural pancreatitis rate $(R R=0.22,95 \%$ $\mathrm{CI}=0.05-1.02)$. EUS-CDS could, therefore, be used as a primary biliary decompression for patients with malignant biliary obstruction. However, SEMS may cause severe complication (i.e., tissue injury) due to stent migration [14]. Currently, LAMS, which was firstly served for drainage of pancreatic fluid collection, is being utilized for biliary drainage to overcome this drawback of SEMS [16].

Nevertheless, EUS-CDS using the SEMS or LAMS is typically a four-step deployment process. When compared to the noncautery enhanced LAMS, ECE-LAMS is an updated technique that improves clinical outcomes attributable to its all-in-one device with simplified stent deployment. A recent meta-analysis indicated that the pooled rates of technical and clinical success of EUS-CDS with LAMS were $94.1 \%$ and $95.9 \%$, respectively [39]. Notably, results from the present study showed that EUSCDS using ECE-LAMS is associated with higher rates of technical success (95.1\%) and clinical success (93.3\%). Furthermore, ECE-LAMS is potentially safer than LAMS to perform EUS-CDS. Its one-step stent deployment reduces adverse event rates. For example, a Japanese observational study reported that the overall adverse events rate of EUS-CDS with LAMS was 36.8\% (7/19) [28], because of its multisteps of deployment procedure. A meta-analysis reporting on EUS-CDS with the use of different stent types revealed an overall adverse event rate of $21.83 \%(43 / 197)$ [40]. Moreover, one recent metaanalysis reported the pooled adverse events rates of EUSCDS was $10.1 \%$ [39]. However, this study was limited by small sample sizes, and inclusion of publications included a mix of LAMS and ECE-LAMS. In the present metaanalysis, we observed that the pooled rate of adverse events following deployment of ECE-LAMS was $15.3 \%$ $\left(95 \% \mathrm{CI}=10.6 \%-21.6 \% ; I^{2}=13 \%\right)$. The pooled rates of short-term and long-term adverse events with ECE-LAMS 
were $4.4 \%$ and $11.3 \%$, respectively. Therefore, the deployment of ECE-LAMS greatly reduces the risk of adverse events.

The pooled rate (11.3\%) of long-term adverse events of ECE-LAMS in the present meta-analysis was mostly attributable to recurrent tumoral obstruction (5.9\%), food impaction (1.0\%), and sump syndrome (1.0\%). Four patients who underwent stent malfunction due to food impaction or sump syndrome from three studies $[23,26,27]$ then used a second stent inserted across the lumen of the ECE-LAMS to resolve this issue. It is noteworthy that a second stent simultaneously positioned inside the ECE-LAMS could prevent such adverse events. Though one recent study was excluded from our analysis due to high rate of lost to followup, the authors found that patients who received a second double-pigtail stent placed through the ECE-LAMS stent showed a significantly lower rate of recurrent biliary obstruction than the cases using ECE-LAMS alone [25].

There are some limitations in the present meta-analysis. As a limitation of our study was the small participant numbers since only six studies with 270 patients were included in the final analysis. Furthermore, the definitions for clinical outcomes were not uniform across the included studies. We cannot completely rule out the possibility of publication bias, but we have attempted to minimize this by using detailed search strategies and generalization of the definitions according to the American Society for Gastrointestinal Endoscopy lexicon [18]. One other limitation was that smaller LAMS (6 $\mathrm{mm}$ and $8 \mathrm{~mm}$ diameter) was unavailable in the USA, which may cause reporting bias [25]. Another limitation was the availability of EUS-CDS is restricted to only a few expert endoscopic centers. This could cause selection bias. However, the present meta-analysis adopted stringent inclusion criteria to ensure appropriate methodologic quality to evaluate the efficacy and safety of EUS-CDS using ECELAMS. Though the abovementioned limitations and biases in published studies warrant caution in interpretation of the results of our study, these results provide implications regarding selection of biliary drainage techniques.

In conclusion, our results suggest that EUS-CDS using ECE-LAMS is effective and safe in patients with biliary obstruction when ERCP failed. Large and randomized controlled observational studies are needed to further refine these findings.

\section{Data Availability}

The datasets used and/or analyzed during the current study are available from the corresponding author on request.

\section{Conflicts of Interest}

The authors declare that they have no conflicts of interest.

\section{Authors' Contributions}

Z.P., R.P., and Y.W. searched the databases. Z.P., S.L., and Y.T. assessed studies and extracted data. Z.P. and S.L. drafted the manuscript. X.L. and W.W. revised the manuscript and language. H.L. contributed to the study design and critical revision of the manuscript. All authors reviewed and approved the manuscript.

\section{Supplementary Materials}

PRISMA checklist is available in the Supplementary Materials. (Supplementary Materials)

\section{References}

[1] M. L. Freeman and N. M. Guda, "ERCP cannulation: a review of reported techniques," Gastrointestinal Endoscopy, vol. 61, no. 1, pp. 112-125, 2005.

[2] A. Bailey, M. Bourke, S. Williams et al., "A prospective randomized trial of cannulation technique in ERCP: effects on technical success and post-ERCP pancreatitis," Endoscopy, vol. 40, no. 4, pp. 296-301, 2008.

[3] L. Enochsson, F. Swahn, U. Arnelo, M. Nilsson, M. Löhr, and G. Persson, "Nationwide, population-based data from 11,074 ERCP procedures from the Swedish registry for gallstone surgery and ERCP," Gastrointestinal Endoscopy, vol. 72, no. 6, pp. 1175-1184, 2010.

[4] R. Tonozuka, T. Itoi, T. Tsuchiya, R. Tanaka, and S. Mukai, "EUS-guided biliary drainage is infrequently used even in high-volume centers of interventional EUS," Gastrointestinal Endoscopy, vol. 84, no. 1, pp. 206-207, 2016.

[5] E. L. A. Artifon, P. Sakai, J. E. M. Cunha et al., "Surgery or endoscopy for palliation of biliary obstruction due to metastatic pancreatic cancer," The American Journal of Gastroenterology, vol. 101, no. 9, pp. 2031-2037, 2006.

[6] S. Nennstiel, A. Weber, G. Frick et al., "Drainage-related complications in percutaneous transhepatic biliary drainage: an analysis over 10 years," Journal of Clinical Gastroenterology, vol. 49, no. 9, pp. 764-770, 2015.

[7] M. Giovannini, V. Moutardier, C. Pesenti, E. Bories, B. Lelong, and J. Delpero, "Endoscopic ultrasound-guided bilioduodenal anastomosis: a new technique for biliary drainage," Endoscopy, vol. 33, no. 10, pp. 898-900, 2001.

[8] A. T. Committee, J. T. Maple, R. Pannala, B. K. Abu Dayyeh, H. R. Aslanian, and B. K. Enestvedt, "Interventional EUS (with videos)," Gastrointestinal Endoscopy, vol. 85, pp. 465481, 2017.

[9] P. Hindryckx, H. Degroote, D. J. Tate, and P. H. Deprez, "Endoscopic ultrasound-guided drainage of the biliary system: techniques, indications and future perspectives," World Journal of Gastrointestinal Endoscopy, vol. 11, no. 2, pp. 103-114, 2019.

[10] R. S. Uemura, M. A. Khan, J. P. Otoch, M. Kahaleh, E. F. Montero, and E. L. A. Artifon, "EUS-guided choledochoduodenostomy versus hepaticogastrostomy," Journal of Clinical Gastroenterology, vol. 52, no. 2, pp. 123-130, 2018.

[11] D. Jain, M. Shah, U. Patel, A. Sharma, and S. Singhal, "Endoscopic ultrasound guided choledocho-enterostomy by using lumen apposing metal stent in patients with failed endoscopic retrograde cholangiopancreatography: a literature review," Digestion, vol. 98, no. 1, pp. 1-10, 2018.

[12] D. H. Park, J. W. Jang, S. S. Lee, D.-W. Seo, S. K. Lee, and M.-H. Kim, "EUS-guided biliary drainage with transluminal stenting after failed ERCP: predictors of adverse events and long-term results," Gastrointestinal Endoscopy, vol. 74, no. 6, pp. 1276-1284, 2011.

[13] J.-M. Dumonceau, A. Tringali, I. Papanikolaou et al., "Endoscopic biliary stenting: indications, choice of stents, and 
results: European society of gastrointestinal endoscopy (ESGE) clinical guideline-updated October 2017," Endoscopy, vol. 50, no. 9, pp. 910-930, 2018.

[14] K. F. Binmoeller, T. DeSimio, and R. Donovan, "Design considerations of the AXIOS stent and electrocautery enhanced delivery system," Techniques and Innovations in Gastrointestinal Endoscopy, vol. 22, no. 1, pp. 3-8, 2020.

[15] N. R. Kalva, V. Vanar, D. Forcione, M. L. Bechtold, and S. R. Puli, "Efficacy and safety of lumen apposing self-expandable metal stents for EUS guided cholecystostomy: a meta-analysis and systematic review," Canadian Journal of Gastroenterology and Hepatology, vol. 2018, Article ID 7070961, 6 pages, 2018.

[16] R. Kunda, M. Pérez-Miranda, U. Will et al., "EUS-guided choledochoduodenostomy for malignant distal biliary obstruction using a lumen-apposing fully covered metal stent after failed ERCP," Surgical Endoscopy, vol. 30, no. 11, pp. 5002-5008, 2016.

[17] A. Liberati, D. G. Altman, J. Tetzlaff et al., "The PRISMA statement for reporting systematic reviews and meta-analyses of studies that evaluate healthcare interventions: explanation and elaboration,” BMJ, vol. 339, no. 1, p. b2700, 2009.

[18] P. B. Cotton, G. M. Eisen, L. Aabakken et al., "A lexicon for endoscopic adverse events: report of an ASGE workshop," Gastrointestinal Endoscopy, vol. 71, no. 3, pp. 446-454, 2010.

[19] R. DerSimonian and N. Laird, "Meta-analysis in clinical trials," Controlled Clinical Trials, vol. 7, no. 3, pp. 177-188, 1986.

[20] J. P. T. Higgins, S. G. Thompson, J. J. Deeks, and D. G. Altman, "Measuring inconsistency in meta-analyses," BMJ, vol. 327, no. 7414, pp. 557-560, 2003.

[21] F. Kanwal and D. White, "“Systematic reviews and metaanalyses" in clinical gastroenterology and hepatology," Clinical Gastroenterology and Hepatology, vol. 10, no. 11, pp. 1184-1186, 2012.

[22] G. H. Guyatt, A. D. Oxman, R. Kunz et al., "GRADE guidelines: 7. Rating the quality of evidence-inconsistency," Journal of Clinical Epidemiology, vol. 64, no. 12, pp. 12941302, 2011.

[23] A. Anderloni, A. Fugazza, E. Troncone et al., "Single-stage EUS-guided choledochoduodenostomy using a lumen-apposing metal stent for malignant distal biliary obstruction," Gastrointestinal Endoscopy, vol. 89, no. 1, pp. 69-76, 2019.

[24] A. Anderloni, M. D. Leo, S. Carrara, A. Fugazza, R. Maselli, and A. Buda, "Endoscopic ultrasound-guided transmural drainage by cautery-tipped lumen-apposing metal stent: exploring the possible indications," Annals of Gastroenterology, vol. 31, pp. 735-741, 2018.

[25] A. H. El Chafic, J. N. Shah, C. Hamerski et al., "EUS-guided choledochoduodenostomy for distal malignant biliary obstruction using electrocautery-enhanced lumen-apposing metal stents: first US, multicenter experience," Digestive Diseases and Sciences, vol. 64, no. 11, pp. 3321-3327, 2019.

[26] J. Jacques, J. Privat, F. Pinard et al., "EUS-guided choledochoduodenostomy by use of electrocautery-enhanced lumen-apposing metal stents: a French multicenter study after implementation of the technique (with video)," Gastrointestinal Endoscopy, vol. 92, no. 1, pp. 134-141, 2020.

[27] J. Jacques, J. Privat, F. Pinard et al., "Endoscopic ultrasoundguided choledochoduodenostomy with electrocautery-enhanced lumen-apposing stents: a retrospective analysis," Endoscopy, vol. 51, no. 6, pp. 540-547, 2019.

[28] T. Tsuchiya, A. Y. B. Teoh, T. Itoi et al., "Long-term outcomes of EUS-guided choledochoduodenostomy using a lumen- apposing metal stent for malignant distal biliary obstruction: a prospective multicenter study," Gastrointestinal Endoscopy, vol. 87, no. 4, pp. 1138-1146, 2018.

[29] E. Rinninella, R. Kunda, M. Dollhopf et al., "EUS-guided drainage of pancreatic fluid collections using a novel lumenapposing metal stent on an electrocautery-enhanced delivery system: a large retrospective study (with video)," Gastrointestinal Endoscopy, vol. 82, no. 6, pp. 1039-1046, 2015.

[30] D. G. Adler, L. J. Taylor, R. Hasan, and A. A. Siddiqui, "A retrospective study evaluating endoscopic ultrasound-guided drainage of pancreatic fluid collections using a novel lumenapposing metal stent on an electrocautery enhanced delivery system," Endoscopic Ultrasound, vol. 6, pp. 389-393, 2017.

[31] A. Mussetto, A. Fugazza, L. Fuccio, O. Triossi, A. Repici, and A. Anderloni, "Current uses and outcomes of lumen-apposing metal stents," Annals of Gastroenterology, vol. 31, pp. 535-540, 2018.

[32] T. Itoi, N. Ikeuchi, R. Tonozuka, S. Mukai, and A. Sofuni, "EUS-guided choledochojejunostomy with a lumen-apposing metal stent in a post-Whipple patient," Gastrointestinal Endoscopy, vol. 81, no. 5, pp. 1259-1260, 2015.

[33] M. Giovannini and E. Bories, "EUS-guided biliary drainage," Gastroenterology Research and Practice, vol. 2012, Article ID 348719, 5 pages, 2012.

[34] D. Makmun, A. Fauzi, M. Abdullah, and A. F. Syam, "The role of EUS-BD in the management of malignant biliary obstruction: the Indonesian perspective," Diagnostic and Therapeutic Endoscopy, vol. 2017, Article ID 4856276, 8 pages, 2017.

[35] J. K. Park, Y. S. Woo, D. H. Noh et al., "Efficacy of EUS-guided and ERCP-guided biliary drainage for malignant biliary obstruction: prospective randomized controlled study," Gastrointestinal Endoscopy, vol. 88, no. 2, pp. 277-282, 2018.

[36] W. H. Paik, T. H. Lee, D. H. Park et al., "EUS-guided biliary drainage versus ERCP for the primary palliation of malignant biliary obstruction: a multicenter randomized clinical trial," American Journal of Gastroenterology, vol. 113, no. 7, pp. 987-997, 2018.

[37] J. Y. Bang, U. Navaneethan, M. Hasan, R. Hawes, and S. Varadarajulu, "Stent placement by EUS or ERCP for primary biliary decompression in pancreatic cancer: a randomized trial (with videos)," Gastrointestinal Endoscopy, vol. 88, no. 1, pp. 9-17, 2018.

[38] K. Bishay, D. Boyne, M. Yaghoobi et al., "Endoscopic ultrasound-guided transmural approach versus ERCP-guided transpapillary approach for primary decompression of malignant biliary obstruction: a meta-analysis," Endoscopy, vol. 51, no. 10, pp. 950-960, 2019.

[39] B. P. Mohan, M. Shakhatreh, R. Garg, S. Ponnada, U. Navaneethan, and D. G. Adler, "Efficacy and safety of endoscopic ultrasound-guided choledochoduodenostomy," Journal of Clinical Gastroenterology, vol. 53, no. 4, pp. 243250, 2019 .

[40] K. Wang, J. Zhu, L. Xing, Y. Wang, Z. Jin, and Z. Li, “Assessment of efficacy and safety of EUS-guided biliary drainage: a systematic review," Gastrointestinal Endoscopy, vol. 83, no. 6, pp. 1218-1227, 2016. 